Volume 70, Number 1, Pages 279-289(2021)

DOI: $10.31801 /$ cfsuasmas. 807169

ISSN 1303-5991 E-ISSN 2618-6470

Received by the editors: October 7, 2020; Accepted: January 22, 2021

\title{
DEFERRED NÖRLUND STATISTICAL RELATIVE UNIFORM CONVERGENCE AND KOROVKIN-TYPE APPROXIMATION THEOREM
}

\author{
Kamil DEMİRCİ, Fadime DİRİK, and Sevda YILDIZ \\ Department of Mathematics, Sinop University, Sinop, TURKEY
}

\begin{abstract}
In this paper, we define the concept of statistical relative uniform convergence of the deferred Nörlund mean and we prove a general Korovkintype approximation theorem by using this convergence method. As an application, we use classical Bernstein polynomials for defining an operator that satisfies our new approximation theorem but does not satisfy the theorem given before. Additionally, we estimate the rate of convergence of approximating positive linear operators by means of the modulus of continuity.
\end{abstract}

\section{Introduction and Preliminaries}

The notion of statistical convergence for the sequences of real numbers was introduced by Steinhaus 17] and Fast [11 independently in the same year. After that, the concept of statistical convergence in approximation theory has been used by Gadjiev and Orhan [12] to prove Korovkin-type approximation theorem. Recent studies on the statistical approximation may be found in the monograph by Anastassiou and Duman 3. Later many researchers have investigated the Korovkin-type approximation theorems for various operators defined on different spaces, for example, the space of all continuous functions, modular space, the space of all Bögel continuous functions, etc. ( $4,6,8,9,13,16,19,20]$ ). In this paper, we define a new concept of statistical relative uniform convergence of the deferred Nörlund mean that improve the deferred Nörlund statistical uniform convergence. Then, we prove a Korovkin type approximation theorem by using this interesting convergence method and show its importance by giving an example. Finally, we study the rate of convergence.

2020 Mathematics Subject Classification. Primary 41A25, 41A36.

Keywords and phrases. Statistical convergence, statistical relative uniform convergence, Nörlund summability, Korovkin-type approximation theorem, modulus of continuity.

\amild@sinop.edu.tr; fdirik@sinop.edu.tr; sevdaorhan@sinop.edu.tr-Corresponding author

(D) 0000-0002-5976-9768; 0000-0002-9316-9037; 0000-0002-4730-2271. 
First, we begin with some definitions and notations.

The natural density of a set $B \subseteq \mathbb{N}$, the set of natural numbers, is defined by

$$
\delta(B):=\lim _{n \rightarrow \infty} \frac{1}{n}|\{k \leq n: k \in B\}|,
$$

provided that the limit on the right-hand side exists $([15])$ where $|B|$ we mean the cardinality of the set $B$.

A sequence $x=\left(x_{n}\right)$ of real numbers is said to be statistically convergent to $L$, if for every $\varepsilon>0$,

$$
\delta\left(\left\{n \in \mathbb{N}:\left|x_{n}-L\right| \geq \varepsilon\right\}\right)=0 .
$$

It is denoted by $s t-\lim _{n \rightarrow \infty} x_{n}=L$.

Now, we remember that the idea of the weighted statistical convergence based upon the deferred Nörlund mean $D_{a}^{b}(N, p, q)$.

Let $\left(a_{n}\right)$ and $\left(b_{n}\right)$ be sequences of nonnegative integers and satisfy the following conditions:

(i) $a_{n}<b_{n}$

(ii) $\lim _{n \rightarrow \infty} b_{n}=\infty$.

The above conditions $(i)$ and $(i i)$ are known as the regularity conditions for the deferred Nörlund mean (see [1]).

Assume that $\left(p_{n}\right)$ and $\left(q_{n}\right)$ are the sequences of nonnegative real numbers such that

$$
P_{n}=\sum_{v=a_{n}+1}^{b_{n}} p_{v} \text { and } Q_{n}=\sum_{v=a_{n}+1}^{b_{n}} q_{v} .
$$

The convolution of the above sequences can be introduced as follows:

$$
R_{a_{n}+1}^{b_{n}}=(P * Q)_{n}=\sum_{v=a_{n}+1}^{b_{n}} p_{v} q_{b_{n}-v} .
$$

In order to define the deferred Nörlund mean $D_{a}^{b}(N, p, q)$, we first set

$$
t_{n}=\frac{1}{R_{a_{n}+1}^{b_{n}}} \sum_{v=a_{n}+1}^{b_{n}} p_{b_{n}-v} q_{v} x_{v}
$$

Then we say that a sequence $x=\left(x_{n}\right)$ is deferred Nörlund statistically summable to $L$ or, briefly, statistically summable $D_{a}^{b}(N, p, q)$, if

$$
s t-\lim _{n \rightarrow \infty} t_{n}=L \text {. }
$$

We denote by $S_{t_{n}}$ the set of all sequences that are $D_{a}^{b}(N, p, q)$ statistically summable.

A sequence $x=\left(x_{n}\right)$ is said to be statistically convergent to $L$ with respect to the deferred Nörlund mean $D_{a}^{b}(N, p, q)$ if, for each $\varepsilon>0$, the following set:

$$
\left\{m \leq R_{a_{n}+1}^{b_{n}}: p_{b_{n}-m} q_{m}\left|x_{m}-L\right| \geq \varepsilon\right\}
$$


has zero deferred Nörlund density, that is,

$$
\lim _{n \rightarrow \infty} \frac{1}{R_{a_{n}+1}^{b_{n}}}\left|\left\{m \leq R_{a_{n}+1}^{b_{n}}: p_{b_{n}-m} q_{m}\left|x_{m}-L\right| \geq \varepsilon\right\}\right|=0 .
$$

In this case, we write $S_{t_{n}}-\lim x_{n}=L$.

Let us turn our attention to the definitions deferred Nörlund statistical pointwise and uniform convergence were given in [18]:

Let $f$ and $f_{n}$, for $\forall n \in \mathbb{N}$, belong to $C(X)$. We denote by $C(X)$ the space of all continuous functions on $X$, which is a compact subset of $\mathbb{R}$. This space is equipped with the supremum norm

$$
\|f\|=\sup _{z \in X}|f(z)|, \quad(f \in C(X)) .
$$

Definition 1. $([18])\left(f_{n}\right)$ is said to be deferred Nörlund statistically pointwise convergent to $f$ on $X$, (i.e., $t_{n}$-statistically pointwise convergent) if for every $\varepsilon>0$ and for each $z \in X$,

$$
\lim _{n \rightarrow \infty} \frac{\Omega_{n}(z, \varepsilon)}{R_{a_{n}+1}^{b_{n}}}=0
$$

where $\Omega_{n}(z, \varepsilon):=\left|\left\{m \leq R_{a_{n}+1}^{b_{n}}: p_{b_{n}-m} q_{m}\left|f_{m}(z)-f(z)\right| \geq \varepsilon\right\}\right|$. In this case we write $f_{n} \rightarrow f\left(t_{n}\right.$-stat-pointwise $)$ on $X$.

In the following, we rewrite the definition of deferred Nörlund statistical uniform convergence given in $[18$ and we extend this definition to the deferred Nörlund statistical relative uniform convergence:

Definition 2. $([18])\left(f_{n}\right)$ is said to be deferred Nörlund statistically uniformly convergent to $f$ on $X$, (i.e., $t_{n}$-statistically uniform convergent) if for every $\varepsilon>0$,

$$
\lim _{n \rightarrow \infty} \frac{\Phi_{n}(\varepsilon)}{R_{a_{n}+1}^{b_{n}}}=0
$$

where $\Phi_{n}(\varepsilon):=\left|\left\{m \leq R_{a_{n}+1}^{b_{n}}: p_{b_{n}-m} q_{m} \sup _{z \in X}\left|f_{m}(z)-f(z)\right| \geq \varepsilon\right\}\right|$. In this case we write $f_{n} \rightrightarrows f\left(t_{n}\right.$-stat-uniform $)$ on $X$.

Let us remind the concept of statistical relative uniform convergence. According to Demirci and Orhan [7, this method is defined as follows:

Definition 3. [7] $\left(f_{n}\right)$ is said to be statistically relatively uniformly convergent to $f$ on $X$ if there exists a scale function $\sigma(z),|\sigma(z)|>0$, such that for every $\varepsilon>0$,

$$
\lim _{n \rightarrow \infty} \frac{K_{n}(\varepsilon)}{n}=0
$$

where $K_{n}(\varepsilon):=\left|\left\{m \leq n: \sup _{z \in X}\left|\frac{f_{m}(z)-f(z)}{\sigma(z)}\right| \geq \varepsilon\right\}\right|$. In this case we write $f_{m} \rightrightarrows f$ $(\sigma ; s t)$ on $X$. 
Actually, the above definitions come from the idea by Duman and Orhan 10 and Karakuş et al. 13. For the purposes of this paper, we now give our new convergence method with the help of Definition 2 and Definition 3.

Definition 4. $\left(f_{n}\right)$ is said to be deferred Nörlund statistically relatively uniformly convergent to $f$ on $X$, (i.e., $t_{n}$-statistically relatively uniformly convergent) if there exists a scale function $\sigma(z),|\sigma(z)|>0$, such that for every $\varepsilon>0$,

$$
\lim _{n \rightarrow \infty} \frac{\Phi_{n}(\varepsilon)}{R_{a_{n}+1}^{b_{n}}}=0
$$

where $\Phi_{n}(\varepsilon):=\left|\left\{m \leq R_{a_{n}+1}^{b_{n}}: p_{b_{n}-m} q_{m} \sup _{z \in X}\left|\frac{f_{m}(z)-f(z)}{\sigma(z)}\right| \geq \varepsilon\right\}\right|$. In this case we write $f_{n} \rightrightarrows f\left(\sigma ; t_{n}\right.$-stat-uniform $)$ on $X$.

Here it is important to say that, $t_{n}$-statistical uniform convergence is the special case of $t_{n}$-statistical relative uniform convergence in which the scale function is a non-zero constant. If the scale function is bounded, then $t_{n}$-statistical relative uniform convergence implies $t_{n}$-statistical relative uniform convergence. But, $t_{n}$-statistical relative uniform convergence does not imply $t_{n}$-statistical uniform convergence, when $\sigma(z)$ is unbounded.

Now, we present the following example showing the importance of our new convergence method.

Example 5. For $p_{n}=1, q_{n}=\frac{n+2}{n+1}, a_{n}=2 n$ and $b_{n}=4 n$, let us define the sequence of continuous real-valued functions $g_{n}:[0,1] \rightarrow \mathbb{R}$ by

$$
g_{n}(z)=\left\{\begin{array}{cc}
\frac{2 n z}{1+n^{2} z^{2}}, & z \in\left[0, \frac{1}{n}\right] \\
0, & z \in\left(\frac{1}{n}, 1\right]
\end{array}\right.
$$

and $g(z)=0$ on $[0,1]$. Since $\left\|g_{n}-g\right\|=\sup _{z \in X}\left|g_{n}(z)-g(z)\right|=1$, then

$p_{b_{n}-m} q_{m} \sup _{z \in X}\left|g_{m}(z)-g(z)\right|=\frac{m+2}{m+1}$. Hence, for $0<\varepsilon \leq 1$, we obtain

$$
\begin{aligned}
& \lim _{n \rightarrow \infty} \frac{1}{R_{a_{n}+1}^{b_{n}}}\left|\left\{m \leq R_{a_{n}+1}^{b_{n}}: p_{b_{n}-m} q_{m} \sup _{z \in X}\left|g_{m}(z)-g(z)\right| \geq \varepsilon\right\}\right| \\
= & \lim _{n \rightarrow \infty} \frac{1}{R_{a_{n}+1}^{b_{n}}}\left|\left\{m \leq R_{a_{n}+1}^{b_{n}}: \frac{m+2}{m+1} \geq \varepsilon\right\}\right| \\
= & 1
\end{aligned}
$$

Therefore, $\left(g_{n}\right)$ is not $t_{n}$-statistically uniformly convergent. Now we get the scale function as follows:

$$
\sigma(z)=\left\{\begin{array}{lc}
\frac{1}{z}, & z \in(0,1] \\
1, & z=0 .
\end{array}\right.
$$


As can be seen from, $\left\|\frac{g_{m}-g}{\sigma}\right\|=\frac{1}{m}$, it is clear that

$$
\begin{gathered}
\lim _{n \rightarrow \infty} \frac{1}{R_{a_{n}+1}^{b_{n}}}\left|\left\{m \leq R_{a_{n}+1}^{b_{n}}: p_{b_{n}-m} q_{m}|| \frac{g_{m}-g}{\sigma} \| \geq \varepsilon\right\}\right| \\
=\lim _{n \rightarrow \infty} \frac{1}{R_{a_{n}+1}^{b_{n}}}\left|\left\{m \leq R_{a_{n}+1}^{b_{n}}: \frac{m+2}{m+1} \cdot \frac{1}{m} \geq \varepsilon\right\}\right|=0, \\
g_{n} \rightrightarrows g=0\left(\sigma ; t_{n} \text {-stat-uniform }\right) \text { on }[0,1] .
\end{gathered}
$$

i.e.,

\section{Korovkin-type APPROXimation theorem}

Let $\left(L_{n}\right)$ be a sequence of positive linear operators on $C(X)$. The study of uniform convergence of $\left(L_{n}(f)\right)$ to a function $f$ by using the test functions defined by $1, z, z^{2}$, was initiated by Korovkin [14 (see, for instance, [2]). Recently, the Korovkin-type theorem has been proved via the concept of statistical convergence in 12 and more recently, the statistical relative Korovkin type approximation theorem has been proved in [7]. In this section, we give the Korovkin-type theorem for sequences of positive linear operators defined on $C(X)$ using the concept of deferred Nörlund statistical relative uniform convergence.

Theorem 6. Let $\left(L_{n}\right)$ be a sequence of positive linear operators acting $C(X)$ into $C(X)$. Then, we have, for all $f \in C(X)$,

$$
L_{n}(f) \rightrightarrows f\left(\sigma ; t_{n} \text {-stat-uniform }\right) \text { on } X,
$$

if and only if

$$
L_{n}\left(f_{i}\right) \rightrightarrows f_{i}\left(\sigma_{i} ; t_{n} \text {-stat-uniform }\right) \text { on } X, i=0,1,2,
$$

where $\left|\sigma_{i}(z)\right|>0, i=0,1,2, \sigma(z):=\max \left\{\sigma_{i}(z): i=0,1,2\right\}$ and $f_{i}(z)=z^{i}, i=$ $0,1,2$.

Proof. Since each of the functions given by $f_{i}(z)=z^{i},(i=0,1,2)$ belong to $C(X)$, the implication $(1) \Rightarrow 2$ is quite obvious. Now, we assume that $(2)$ holds. Let $f$ belongs to $C(X)$ and $z \in X$ fixed. Then there exists a constant $\kappa>0$ such that,

$$
|f(z)| \leq \kappa
$$

which ensure that

$$
|f(u)-f(z)| \leq 2 \kappa
$$

From the continuity of $f$, for a given $\varepsilon>0$, there exists $\delta>0$ such that

$$
|f(u)-f(z)|<\varepsilon \text { whenever }|u-z|<\delta
$$

for every $u \in X$. Now let $\phi(u, z)=(u-z)^{2}$. If $|u-z| \geq \delta, u \in X$, we get

$$
|f(u)-f(z)|<\frac{2 \kappa}{\delta^{2}} \phi(u, z) \text {. }
$$


From (3) and (4), we can see that

$$
|f(u)-f(z)|<\varepsilon+\frac{2 \kappa}{\delta^{2}} \phi(u, z),
$$

i.e.,

$$
-\varepsilon-\frac{2 \kappa}{\delta^{2}} \phi(u, z) \leq f(u)-f(z) \leq \varepsilon+\frac{2 \kappa}{\delta^{2}} \phi(u, z) .
$$

Since the positive linear operator $L_{n}(1 ; z)$ is monotone, by applying this operator to the inequality in (5), we have

$$
\begin{aligned}
L_{n}(1 ; z)\left(-\varepsilon-\frac{2 \kappa}{\delta^{2}} \phi(u, z)\right) & \leq L_{n}(1 ; z)(f(u)-f(z)) \\
& \leq L_{n}(1 ; z)\left(\varepsilon+\frac{2 \kappa}{\delta^{2}} \phi(u, z)\right) .
\end{aligned}
$$

Then, we obtain the following inequality:

$$
\begin{aligned}
-\varepsilon L_{n}(1 ; z)-\frac{2 \kappa}{\delta^{2}} L_{n}(\phi(u, z) ; z) & \leq L_{n}(f ; z)-f(z) L_{n}(1 ; z) \\
& \leq \varepsilon L_{n}(1 ; z)+\frac{2 \kappa}{\delta^{2}} L_{n}(\phi(u, z) ; z) .
\end{aligned}
$$

Since

$$
L_{n}(f ; z)-f(z)=\left[L_{n}(f ; z)-f(z) L_{n}(1 ; z)\right]+f(z)\left[L_{n}(1 ; z)-1\right],
$$

we apply the equality (8) in (7), it can be easily seen that,

$$
L_{n}(f ; z)-f(z) \leq \varepsilon L_{n}(1 ; z)+\frac{2 \kappa}{\delta^{2}} L_{n}(\phi(u, z) ; z)+f(z)\left[L_{n}(1 ; z)-1\right] .
$$

Now we calculate the term of $L_{n}(\phi(u, z) ; z)$. We can write the following:

$$
\begin{aligned}
L_{n}(\phi(u, z) ; z) & =L_{n}\left((u-z)^{2} ; y\right) \\
& =L_{n}\left(u^{2}-2 z u+z^{2} ; z\right) \\
& =L_{n}\left(u^{2} ; z\right)-2 z L_{n}(u ; z)+z^{2} L_{n}(1 ; z) \\
& =\left[L_{n}\left(u^{2} ; z\right)-z^{2}\right]-2 z\left[L_{n}(u ; z)-z\right]+z^{2}\left[L_{n}(1 ; z)-1\right] .
\end{aligned}
$$

By using $(9)$, we have

$$
\begin{aligned}
L_{n}(f ; z)-f(z) \leq & \varepsilon L_{n}(1 ; z)+f(z)\left[L_{n}(1 ; z)-1\right] \\
& +\frac{2 \kappa}{\delta^{2}}\left\{\left[L_{n}\left(u^{2} ; z\right)-z^{2}\right]-2 z\left[L_{n}(u ; z)-z\right]+z^{2}\left[L_{n}(1 ; z)-1\right]\right\} \\
= & \varepsilon+\varepsilon\left[L_{n}(1 ; z)-1\right]+f(z)\left[L_{n}(1 ; z)-1\right] \\
& +\frac{2 \kappa}{\delta^{2}}\left\{\left[L_{n}\left(u^{2} ; z\right)-z\right]-2 z\left[L_{n}(u ; z)-z\right]+z^{2}\left[L_{n}(1 ; z)-1\right]\right\} .
\end{aligned}
$$

Since $\varepsilon>0$ is arbitrary, we can write

$$
\left|L_{n}(f ; z)-f(z)\right| \leq \digamma\left\{\left|L_{n}(1 ; z)-1\right|+\left|L_{n}(u ; z)-z\right|+\left|L_{n}\left(u^{2} ; z\right)-z^{2}\right|\right\}
$$


where $\digamma:=\max \left\{\varepsilon+\kappa+\frac{2 \kappa}{\delta^{2}}\left\|f_{2}\right\|, \frac{4 \kappa}{\delta^{2}}\left\|f_{1}\right\|, \frac{2 \kappa}{\delta^{2}}\right\}$. Let $\sigma(z):=\max \left\{\sigma_{i}(z): i=0,1,2\right\}$ and $\left|\sigma_{i}(z)\right|>0: i=0,1,2$. Then, we get

$$
\begin{aligned}
\left|\frac{L_{n}(f ; z)-f(z)}{\sigma(z)}\right| \leq & \digamma\left\{\left|\frac{L_{n}\left(f_{0} ; z\right)-f_{0}(z)}{\sigma_{0}(z)}\right|+\left|\frac{L_{n}\left(f_{1} ; z\right)-f_{1}(z)}{\sigma_{1}(z)}\right|\right. \\
& \left.+\left|\frac{L_{n}\left(f_{2} ; z\right)-f_{2}(z)}{\sigma_{2}(z)}\right|\right\}
\end{aligned}
$$

Taking supremum on $X$, we get

$$
\left\|\frac{L_{n}(f)-f}{\sigma}\right\| \leq \digamma\left\{\left\|\frac{L_{n}\left(f_{0}\right)-f_{0}}{\sigma_{0}}\right\|+\left\|\frac{L_{n}\left(f_{1}\right)-f_{1}}{\sigma_{1}}\right\|+\left\|\frac{L_{n}\left(f_{2}\right)-f_{2}}{\sigma_{2}}\right\|\right\} .
$$

Now, for a given $r>0$, define the following sets:

$$
\Phi_{n}(r):=\left|\left\{m \leq R_{a_{n}+1}^{b_{n}}: p_{b_{n}-m} q_{m}\left\|\frac{L_{m}(f)-f}{\sigma}\right\| \geq r\right\}\right|
$$

and

$$
\Phi_{n, i}\left(\frac{r}{3 \digamma}\right):=\left|\left\{m \leq R_{a_{n}+1}^{b_{n}}: p_{b_{n}-m} q_{m}\left\|\frac{L_{m}\left(f_{i}\right)-f_{i}}{\sigma_{i}}\right\| \geq \frac{r}{3 \digamma}\right\}\right|,(i=0,1,2) .
$$

It is easy to see that $\frac{\Phi_{n}(r)}{R_{a_{n}+1}^{b_{n}}} \leq \sum_{i=0}^{2} \frac{\Phi_{n, j}\left(\frac{r}{3 \digamma}\right)}{R_{a_{n}+1}^{b_{n}}}$. Then using the hypothesis 2 and considering Definition 3 , the right hand side of 10 tend to zero as $n \rightarrow \infty$. Hence, the proof is completed.

Example 7. Let $X=[0,1]$. For $p_{n}=1, q_{n}=\frac{n+2}{n+1}, a_{n}=2 n$ and $b_{n}=4 n$, we first consider the Bernstein polynomials:

$$
B_{n}(f ; z)=\sum_{k=0}^{n} f\left(\frac{k}{n}\right)\left(\begin{array}{l}
n \\
k
\end{array}\right) z^{k}(1-z)^{n-k}, f \in C[0,1] .
$$

It is known that

$$
\begin{aligned}
B_{n}\left(f_{i} ; z\right) & =f_{i}(z), i=0,1 \\
B_{n}\left(f_{2} ; z\right) & =f_{2}(z)+\frac{z(1-z)}{n} .
\end{aligned}
$$

Using this polynomial, we define a sequence of positive linear operators $D_{n}: C[0,1] \rightarrow C[0,1]$ as follows:

$$
D_{n}(f ; z)=\left(1+g_{n}(z)\right) B_{n}(f ; z), z \in[0,1] \text { and } f \in C[0,1] .
$$

If we choose the sequence $\left(g_{n}(z)\right)$ of functions as we considered in Example 5 , then we can see that

$$
\begin{aligned}
D_{n}\left(f_{0} ; z\right) & =\left(1+g_{n}(z)\right) f_{0}(z) \\
D_{n}\left(f_{1} ; z\right) & =\left(1+g_{n}(z)\right) f_{1}(z) \\
\left.D_{n}\left(f_{2} ; z\right)\right) & =\left(1+g_{n}(z)\right)\left[f_{2}(z)+\frac{z(1-z)}{n}\right] .
\end{aligned}
$$


Now, we consider the scale function as follows:

$$
\sigma(z)=\left\{\begin{array}{lc}
\frac{1}{z}, & z \in(0,1] \\
1, & z=0
\end{array}\right.
$$

Hence, after some simple calculations, it can be easily seen that,

$$
\begin{aligned}
\left\|\frac{D_{n}\left(f_{0}\right)-f_{0}}{\sigma}\right\| & =\left\|\frac{g_{n}}{\sigma}\right\| \\
\left\|\frac{D_{n}\left(f_{1}\right)-f_{1}}{\sigma}\right\| & \leq\left\|\frac{g_{n}}{\sigma}\right\| \\
\left\|\frac{D_{n}\left(f_{2}\right)-f_{2}}{\sigma}\right\| & =\sup _{z \in[0,1]}\left|\frac{\left(1+g_{n}(z)\right)\left[f_{2}(z)+\frac{z(1-z)}{n}\right]-f_{2}(z)}{\sigma(z)}\right| \\
& =\sup _{z \in[0,1]}\left|\frac{\frac{z(1-z)}{n}+g_{n}(z) f_{2}(z)+\frac{z(1-z)}{n} g_{n}(z)}{\sigma(z)}\right| \\
& \leq\left\|\frac{g_{n}}{\sigma}\right\|+\frac{1}{4 n}\left\|\frac{1}{\sigma}\right\|+\frac{1}{4 n}\left\|\frac{g_{n}}{\sigma}\right\| \\
& \leq 2\left\|\frac{g_{n}}{\sigma}\right\|+\frac{1}{n} .
\end{aligned}
$$

Since

$$
g_{n} \rightrightarrows g=0\left(\sigma ; t_{n} \text {-stat-uniform }\right) \text { on }[0,1] \text { and } \frac{1}{n} \rightarrow 0,
$$

we conclude that

$$
D_{n}\left(f_{i}\right) \rightrightarrows f_{i}\left(\sigma ; t_{n} \text {-stat-uniform }\right) \text { on } X, i=0,1,2 .
$$

Then, by our main theorem, Theorem 6, we have

$$
D_{n}(f) \rightrightarrows f\left(\sigma ; t_{n} \text {-stat-uniform }\right) \text { on } X \text {. }
$$

Furthermore, since the sequence $\left(g_{n}\right)$ of functions on $[0,1]$ is not $t_{n}$-statistically uniformly convergent to the function $g=0$ on the interval $[0,1]$, we can say that Korovkin-type approximation theorem given via deferred Nörlund statistical uniform convergence does not hold for our operators defined by (11).

\section{Rate of Convergence}

In this section, we compute the rate of the deferred Nörlund statistical relative uniform convergence of a sequence of positive linear operators defined on $C(X)$ by means of the modulus of continuity.

Now we recall the concept of modulus of continuity. For $f \in C(X)$, the modulus of continuity of $f$ is defined by

$$
\omega(f ; \delta)=\sup _{|u-z| \leq \delta, u, z \in X}|f(u)-f(z)| .
$$


It is also well known that for any $\delta>0$ and each $u, z \in X$

$$
|f(u)-f(z)| \leq \omega(f ; \delta)\left(\frac{|u-z|}{\delta}+1\right) .
$$

Now, we state and prove the following theorem.

Theorem 8. Let $\left(L_{n}\right)$ be a sequence of positive linear operators acting $C(X)$ into $C(X)$. Assume that the following conditions hold:

(i) $L_{n}\left(f_{0}\right) \rightrightarrows f_{0}\left(\sigma_{0} ; t_{n}\right.$-stat-uniform $)$ on $X$,

(ii) $\omega\left(f, \alpha_{n}\right) \rightrightarrows 0\left(\sigma_{1} ; t_{n}\right.$-stat-uniform $)$ on $X$,

where $\alpha_{n}(y)=\sqrt{\left\|L_{n}(\varphi(., z))\right\|}$ with $\varphi(u, z)=(u-z)^{2},\left|\sigma_{i}(z)\right|>0, i=0,1$ and $\sigma(z):=\max \left\{\sigma_{i}(z): i=0,1\right\}$.

Then we have, for all $f \in C(X)$,

$$
L_{n}(f) \rightrightarrows f\left(\sigma ; t_{n} \text {-stat-uniform }\right) \text { on } X .
$$

Proof. Let $f \in C(X)$ and $z \in X$. It is known that $([2])$,

$$
\begin{aligned}
\left|L_{n}(f ; z)-f(z)\right| \leq & L_{n}(|f(u)-f(z)| ; z)+\kappa\left|L_{n}\left(f_{0} ; z\right)-f_{0}(z)\right| \\
\leq & L_{n}\left(\omega(f ; \delta)\left(\frac{|u-z|}{\delta}+1\right) ; z\right)+\kappa\left|L_{n}\left(f_{0} ; z\right)-f_{0}(z)\right| \\
\leq & L_{n}\left(\omega(f ; \delta)\left(\frac{(u-z)^{2}}{\delta^{2}}+1\right) ; z\right)+\kappa\left|L_{n}\left(f_{0} ; z\right)-f_{0}(z)\right| \\
= & \kappa\left|L_{n}\left(f_{0} ; z\right)-f_{0}(z)\right|+\frac{\omega(f ; \delta)}{\delta^{2}} L_{n}\left((u-z)^{2} ; z\right) \\
& +\omega(f ; \delta) L_{n}\left(f_{0} ; z\right) \\
\leq & \kappa\left|L_{n}\left(f_{0} ; z\right)-f_{0}(z)\right|+\frac{\omega(f ; \delta)}{\delta^{2}} L_{n}\left((u-z)^{2} ; z\right) \\
& +\omega(f ; \delta)\left|L_{n}\left(f_{0} ; z\right)-f_{0}(z)\right|+\omega(f ; \delta)
\end{aligned}
$$

where $\kappa:=\|f\|$ and $\varphi(u, z)=(u-z)^{2}$. Then, taking supremum on $X$, we obtain

$$
\begin{aligned}
\left\|\frac{L_{n}(f)-f}{\sigma}\right\| \leq & \kappa\left\|\frac{L_{n}\left(f_{0}\right)-f_{0}}{\sigma_{0}}\right\|+2\left\|\frac{\omega\left(f, \alpha_{n}\right)}{\sigma_{1}}\right\| \\
& +\left\|\frac{L_{n}\left(f_{0}\right)-f_{0}}{\sigma_{0}}\right\|\left\|\frac{\omega\left(f, \alpha_{n}\right)}{\sigma_{1}}\right\|
\end{aligned}
$$

where $\delta:=\alpha_{n}(y)=\sqrt{\left\|L_{n}(\varphi(., z))\right\|},\left|\sigma_{i}(z)\right|>0, i=0,1$ and $\sigma(z):=\max \left\{\sigma_{i}(z): i=0,1\right\}$.

Now, for a given $r>0$, define the following sets:

$$
\Phi_{n}(r):=\left|\left\{m \leq R_{a_{n}+1}^{b_{n}}: p_{b_{n}-m} q_{m}\left\|\frac{L_{m}(f)-f}{\sigma}\right\| \geq r\right\}\right|
$$

and

$$
\Phi_{n, 0}\left(\frac{r}{3}\right):=\left|\left\{m \leq R_{a_{n}+1}^{b_{n}}: p_{b_{n}-m} q_{m}\left\|\frac{L_{m}\left(f_{0}\right)-f_{0}}{\sigma_{0}}\right\|\left\|\frac{\omega\left(f, \alpha_{m}\right)}{\sigma_{1}}\right\| \geq \frac{r}{3}\right\}\right|,
$$




$$
\begin{aligned}
\Phi_{n, 1}\left(\frac{r}{3 \kappa}\right): & =\left|\left\{m \leq R_{a_{n}+1}^{b_{n}}: p_{b_{n}-m} q_{m}\left\|\frac{L_{m}\left(f_{0}\right)-f_{0}}{\sigma_{0}}\right\| \geq \frac{r}{3 \kappa}\right\}\right|, \\
\Phi_{n, 2}\left(\frac{r}{6}\right): & =\left|\left\{m \leq R_{a_{n}+1}^{b_{n}}: p_{b_{n}-m} q_{m}\left\|\frac{\omega\left(f, \alpha_{m}\right)}{\sigma_{1}}\right\| \geq \frac{r}{6}\right\}\right|,
\end{aligned}
$$

Then, we can get

$$
\frac{\Phi_{n}(r)}{R_{a_{n}+1}^{b_{n}}} \leq \frac{\Phi_{n, 0}\left(\frac{r}{3}\right)}{R_{a_{n}+1}^{b_{n}}}+\frac{\Phi_{n, 1}\left(\frac{r}{3 \kappa}\right)}{R_{a_{n}+1}^{b_{n}}}+\frac{\Phi_{n, 2}\left(\frac{r}{6}\right)}{R_{a_{n}+1}^{b_{n}}} .
$$

Now, we also define the following sets:

$$
\begin{aligned}
& \Phi_{n, 3}\left(\sqrt{\frac{r}{3}}\right):=\left|\left\{m \leq R_{a_{n}+1}^{b_{n}}: p_{b_{n}-m} q_{m}\left\|\frac{\omega\left(f, \alpha_{m}\right)}{\sigma_{1}}\right\| \geq \sqrt{\frac{r}{3}}\right\}\right|, \\
& \Phi_{n, 4}\left(\sqrt{\frac{r}{3}}\right):=\left|\left\{m \leq R_{a_{n}+1}^{b_{n}}: p_{b_{n}-m} q_{m}\left\|\frac{L_{m}\left(f_{0}\right)-f_{0}}{\sigma_{0}}\right\| \geq \sqrt{\frac{r}{3}}\right\}\right| .
\end{aligned}
$$

We can easily see that

which gives

$$
\frac{\Phi_{n, 0}\left(\frac{r}{3}\right)}{R_{a_{n}+1}^{b_{n}}} \leq \frac{\Phi_{n, 3}\left(\sqrt{\frac{r}{3}}\right)}{R_{a_{n}+1}^{b_{n}}}+\frac{\Phi_{n, 4}\left(\sqrt{\frac{r}{3}}\right)}{R_{a_{n}+1}^{b_{n}}}
$$

$$
\frac{\Phi_{n}(r)}{R_{a_{n}+1}^{b_{n}}} \leq \frac{\Phi_{n, 1}\left(\frac{r}{3 \kappa}\right)}{R_{a_{n}+1}^{b_{n}}}+\frac{\Phi_{n, 2}\left(\frac{r}{6}\right)}{R_{a_{n}+1}^{b_{n}}}+\frac{\Phi_{n, 3}\left(\sqrt{\frac{r}{3}}\right)}{R_{a_{n}+1}^{b_{n}}}+\frac{\Phi_{n, 4}\left(\sqrt{\frac{r}{3}}\right)}{R_{a_{n}+1}^{b_{n}}} .
$$

Then the hypothesis $(i)$ and $(i i)$ leads us to the conclusion that the right hand side of (13) tend to zero as $n \rightarrow \infty$. The proof is completed.

Declaration of Competing Interests The authors declare that they have no conflict of interest.

Authors Contribution Statement All authors have contributed sufficiently in the planning, execution, or analysis of this study to be included as authors. All authors read and approved the final manuscript.

Acknowledgements This research was supported by Sinop University Scientific Research Coordination Unit, Project N. FEF-1901-18-25

\section{REFERENCES}

[1] Agnew, R.P., On deferred Cesàro means, Ann. Math., 33 (1932), 413-421. https://doi.org/10.2307/1968524

[2] Altomare, F. and Campiti, M., Korovkin-Type Approximation Theory and Its Applications, de Gruyter Stud. Math. 17, Walter de Gruyter, Berlin, 1994. https://doi.org/10.1515/9783110884586 
[3] Anastassiou G. A. and Duman, O., Towards Intelligent Modeling: Statistical Approximation Theory, Intelligent Systems Reference Library, 14, Springer-Verlag, Berlin Heidelberg, 2011. https://doi.org/10.1007/978-3-642-19826-7

[4] Bardaro, C., Boccuto, A., Demirci, K., Mantellini, I., Orhan, S., Triangular $A$-statistical approximation by double sequences of positive linear operators, Results. Math., 68 (2015), 271-291. https://doi.org/10.1007/s00025-015-0433-7

[5] Bardaro, C., Boccuto, A., Demirci, K., Mantellini, I., Orhan, S., Korovkin-type theorems for modular $\Psi-A$-statistical convergence, J. Funct. Spaces, Article ID 160401 (2015), p. 11. https://doi.org/10.1155/2015/160401

[6] Bardaro, C. and Mantellini, I., Korovkin's theorem in modular spaces, Commentationes Math., 47 (2007), 239-253. https://doi.org/10.14708/cm.v47i2.5253

[7] Demirci, K. and Orhan, S., Statistically relatively uniform convergence of positive linear operators, Results. Math., 69 (2016), 359-367. https://doi.org/10.1007/s00025-015-0484-9

[8] Dirik, F. and Demirci, K., Korovkin type approximation theorem for functions of two variables in statistical sense, Turk. J. Math., 34 (2010), 73-83. https://doi.org/10.3906/mat-0802-21

[9] Dirik, F. and Demirci, K., Approximation in Statistical Sense to $n$-variate $B$ Continuous Functions by Positive Linear Operators, Math. Slovaca, 60 (2010), 877-886. https://doi.org/10.2478/s12175-010-0054-2

[10] Duman, O. and Orhan, C., $\mu$-statistically convergent function sequences, Czechoslovak Math. J., 54 (129) (2004), 413-422. https://doi.org/10.1023/B:CMAJ.0000042380.31622.39

[11] Fast, H., Sur la convergence statistique, Colloq. Math., 2 (1951), 241-244. https://doi.org/10.4064/cm-2-3-4-241-244

[12] Gadjiev, A.D. and Orhan, C., Some approximation theorems via statistical convergence, Rocky Mountain J. Math., 32 (2002), 129-138. https://www.jstor.org/stable/44238888

[13] Karakuş, S., Demirci, K. and Duman, O., Equi-statistical convergence of positive linear operators, J. Math. Anal. Appl., 339 (2008), 1065-1072. https://doi.org/10.1016/j.jmaa.2007.07.050

[14] Korovkin, P.P., Linear Operators and Approximation Theory, Hindustan Publ. Co., Delhi, 1960.

[15] Niven, I. and Zuckerman, H.S., An Introduction to the Theory of Numbers, John Wiley and Sons, Fourt Ed., New York, 1980.

[16] Söylemez, D. and Ünver, M., Korovkin type theorems for Cheney-Sharma operators via summability methods, Results. Math., 72(3) (2017) 1601-1612. https://doi.org/10.1007/s00025017-0733-1

[17] Steinhaus, H., Sur la convergence ordinaire et la convergence asymtotique, Colloq. Math., 2 (1951), 73-74

[18] Srivastava, H. M. Bidu Bhusan Jena, Susanta Kumar Paikray, Misra, U. K., Generalized equistatistical convergence of the deferred Nörlund summability and its applications to associated approximation theorems, RACSAM, 112 (2018), 1487-1501. https://doi.org/10.1007/s13398017-0442-3

[19] Taş, E. and Yurdakadim, T., Approximation by positive linear operators in modular spaces by power series method, Positivity, 21(4) (2017) 1293-1306. https://doi.org/10.1007/s11117017-0467-z

[20] Yilmaz, B., Demirci, K., Orhan, S., Relative Modular Convergence of Positive Linear Operators, Positivity, 20 (2016), 565-577. https://doi.org/10.1007/s11117-015-0372-2 